\title{
Electromagnetic parameter retrieval from inhomogeneous metamaterials
}

\author{
D. R. Smith, ${ }^{1,2, *}$ D. C. Vier, ${ }^{2}$ Th. Koschny, ${ }^{3,4}$ and C. M. Soukoulis ${ }^{3,4}$ \\ ${ }^{1}$ Department of Electrical and Computer Engineering, Duke University, Box 90291, Durham, North Carolina 27708, USA \\ ${ }^{2}$ Department of Physics, University of California, San Diego, La Jolla, California 92093, USA \\ ${ }^{3}$ Ames Laboratory and Department of Physics and Astronomy, Iowa State University, Ames, Iowa 50011, USA \\ ${ }^{4}$ Foundation for Research and Technology Hellas (FORTH), 71110 Heraklion, Crete, Greece
}

(Received 21 November 2004; published 22 March 2005)

\begin{abstract}
We discuss the validity of standard retrieval methods that assign bulk electromagnetic properties, such as the electric permittivity $\varepsilon$ and the magnetic permeability $\mu$, from calculations of the scattering $(S)$ parameters for finite-thickness samples. $S$-parameter retrieval methods have recently become the principal means of characterizing artificially structured metamaterials, which, by nature, are inherently inhomogeneous. While the unit cell of a metamaterial can be made considerably smaller than the free space wavelength, there remains a significant variation of the phase across the unit cell at operational frequencies in nearly all metamaterial structures reported to date. In this respect, metamaterials do not rigorously satisfy an effective medium limit and are closer conceptually to photonic crystals. Nevertheless, we show here that a modification of the standard $S$-parameter retrieval procedure yields physically reasonable values for the retrieved electromagnetic parameters, even when there is significant inhomogeneity within the unit cell of the structure. We thus distinguish a metamaterial regime, as opposed to the effective medium or photonic crystal regimes, in which a refractive index can be rigorously established but where the wave impedance can only be approximately defined. We present numerical simulations on typical metamaterial structures to illustrate the modified retrieval algorithm and the impact on the retrieved material parameters. We find that no changes to the standard retrieval procedures are necessary when the inhomogeneous unit cell is symmetric along the propagation axis; however, when the unit cell does not possess this symmetry, a modified procedure-in which a periodic structure is assumed - is required to obtain meaningful electromagnetic material parameters.
\end{abstract}

DOI: 10.1103/PhysRevE.71.036617

PACS number(s): 41.20.- q, 42.70.- a

\section{INTRODUCTION}

\section{A. Effective media}

It is conceptually convenient to replace a collection of scattering objects by a homogeneous medium, whose electromagnetic properties result from an averaging of the local responding electromagnetic fields and current distributions. Ideally, there would be no distinction in the observed electromagnetic response of the hypothetical continuous material versus that of the composite it replaces. This equivalence can be readily achieved when the applied fields are static or have spatial variation on a scale significantly larger than the scale of the local inhomogeneity, in which case the composite is said to form an effective medium.

The electromagnetic properties of an inhomogeneous composite can be determined exactly by solving Maxwell's equations, which relate the local electric and magnetic fields to the local charge and current densities. When the particular details of the inhomogeneous structure are unimportant to the behavior of the relevant fields of interest, the local field, charge, and current distributions are averaged, yielding the macroscopic form of Maxwell's equations [1]. To solve this set of equations, a relationship must be assumed that relates the four macroscopic field vectors that arise from the averaging-or homogenization-procedure. It is here that the electric permittivity $(\varepsilon)$ and the magnetic permeability

\footnotetext{
*Email address: drsmith@ee.duke.edu
}

$(\boldsymbol{\mu})$ tensors are typically defined, which encapsulate the specific local details of the composite medium [2-5].

Depending on the symmetry and complexity of the scattering objects that comprise the composite medium, the $\boldsymbol{\varepsilon}$ and $\boldsymbol{\mu}$ tensors may not provide sufficient information to obtain a solution from Maxwell's equations, and additional electromagnetic material parameters must be introduced [6]. Such media, including chiral and bianisotropic, can couple polarization states and are known to host a wide array of wave propagation and other electromagnetic phenomena [7]. We exclude these more complicated materials from our discussion here, however, focusing our attention on linear, passive media whose electromagnetic properties can be entirely specified by the $\boldsymbol{\varepsilon}$ and $\boldsymbol{\mu}$ tensors. Moreover, we restrict our characterization of the material to one of its principal axes, and furthermore assume the probing wave is linearly polarized; thus, only the two scalar components $\varepsilon$ and $\mu$ are relevant.

The analytical approaches used in effective medium theory offer important physical insight into the nature of the electromagnetic response of a composite; however, analytical techniques become increasingly difficult to apply in cases where the scattering elements have complex geometry. As an alternative for such composites, a numerical approach is feasible in which the local electromagnetic fields of a structure are calculated by direct integration of Maxwell's equations, and an averaging procedure applied to define the macroscopic fields and material parameters $[2,8,9]$. Such an approach is feasible for simulations, but does not extend to 
experimental measurements, where retrieval techniques are of prime importance for characterization of structures.

Distilling the complexity of an artificial medium into bulk electromagnetic parameters is of such advantage that homogenization methods have been extended beyond the traditional regime where effective medium theories would be considered valid $[10,11]$. The derived electromagnetic parameters, even if approximate, can be instrumental in the design of artificial materials and in the interpretation of their scattering properties. Indeed, much of the recent activity in electromagnetic composites has demonstrated that scattering elements whose dimensions are an appreciable fraction of a wavelength $(>\lambda / 10)$ can be described in practice by the effective medium parameters $\varepsilon$ and $\mu$. In particular, artificial materials, or metamaterials, with negative refractive index have been designed and characterized following the assumption that some form of effective medium theory applies [12]. These metamaterials have successfully been shown to interact with electromagnetic radiation in the same manner as would homogeneous materials with equivalent material parameters [13].

\section{B. Periodic structures}

While the metamaterials introduced over the past several years have been mostly represented in the literature as examples of effective media, their actual design typically consists of scattering elements arranged or patterned periodically. This method of forming a metamaterial is convenient from the standpoint of design and analysis, because a complete numerical solution of Maxwell's equations can be obtained from consideration of one unit cell of a periodic structure $[14,15]$. The electromagnetic structure associated with periodic systems-or photonic crystals-possesses numerous modes, especially toward higher frequencies, that are typically summarized in band diagrams. The band diagram enumerates Bloch waves rather than plane waves, as the Bloch wave represents the solution to Maxwell's equations for systems with $\varepsilon$ and $\mu$ having spatial periodicity [14].

The photonic crystal description of a periodic structure is valid for all ratios of the wavelength relative to the scale of inhomogeneity. But when the wavelength is very large relative to the inhomogeneity, then it can be expected that an effective medium description should also be valid. It might also be expected that there should exist a transitional regime between the effective medium and the photonic crystal descriptions of a periodic structure, where definitions of $\varepsilon$ and $\mu$ can be applied that are approximate but yet have practical utility. It is this regime into which most of the recently demonstrated metamaterial structures fall. The metamaterial used to demonstrate negative refraction in 2001 , for example, had a unit cell dimension $d$ that was roughly one-sixth the free space wavelength [13], meaning that the physically relevant optical path length $k d=2 \pi d / \lambda \sim 1$. The validity of ascribing values of $\varepsilon$ and $\mu$ to periodic systems for which $k d$ is not small relative to unity was explored by Kyriazidou et al., who concluded that the effective response functions for periodic structures can be extended significantly beyond the traditional limits of effective medium theory, even to the point where the free space wavelength is on the order of the unit cell dimensions [11].

Similar to [11], we present here an algorithmic approach for the assignment of effective medium parameters to a periodic structure. Our approach makes use of the scattering parameters determined for a finite-thickness, planar slab of the inhomogeneous structure to be characterized. Knowing the phases and amplitudes of the waves transmitted and reflected from the slab, we can retrieve values for the complex refractive index $n$ and wave impedance $z$. We find that a valid effective refractive index can always be obtained for the inhomogeneous, periodic structure. A wave impedance can also be assigned to the composite; however, the impedance value will depend on the termination of the unit cell. In particular, for structures that are not symmetric along the wave propagation direction, two different values of impedance are retrieved corresponding to the two incident directions of wave propagation. This ambiguity in the impedance leads to a fundamental ambiguity in the definitions of $\varepsilon$ and $\mu$, which increases as the ratio of unit cell dimension to wavelength increases. Nevertheless, our analysis shows that the methods used to date to characterize metamaterials are approximately accurate and, with slight modification, are sufficient to be used to generate initial designs of metamaterial structures.

\section{RETRIEVAL METHODS}

\section{A. $S$-parameter retrieval}

If an inhomogeneous structure can be replaced conceptually by a continuous material, there should be no difference in the scattering characteristics between the two. A procedure, then, for the assignment of effective material parameters to an inhomogeneous structure consists of comparing the scattered waves (i.e., the complex transmission and reflection coefficients, or $S$ parameters) from a planar slab of the inhomogeneous material to those scattered from a hypothetical continuous material. Assuming the continuous material is characterized by an index $n$ and an impedance $z$, relatively simple analytic expressions can be found relating $n$ and $z$ of a slab to the $S$ parameters. The inversion of $S$ parameters is an established method for the experimental characterization of unknown materials $[16,17]$. An $S$-parameter measurement is depicted in Fig. 1(a).

The thickness of the slab, $L$, is irrelevant to the retrieved material parameters, which are intrinsic properties of the material. It is thus advantageous when characterizing a material to use as thin a sample as possible-ideally, to be in the limit $k L \ll 1$. Because metamaterials are formed from discrete elements whose periodicity places a strict minimum on the thickness of a sample, we assume that the thickness used hereon represents both one unit cell of the sample as well as the total slab thickness. That is, we set $L=d$. Note that while measurements on a single thickness of sample cannot uniquely determine the material parameters of the sample, it is usually the case that some knowledge regarding the expected sample properties is available beforehand to help identify the most appropriate material parameter set. 




(a)
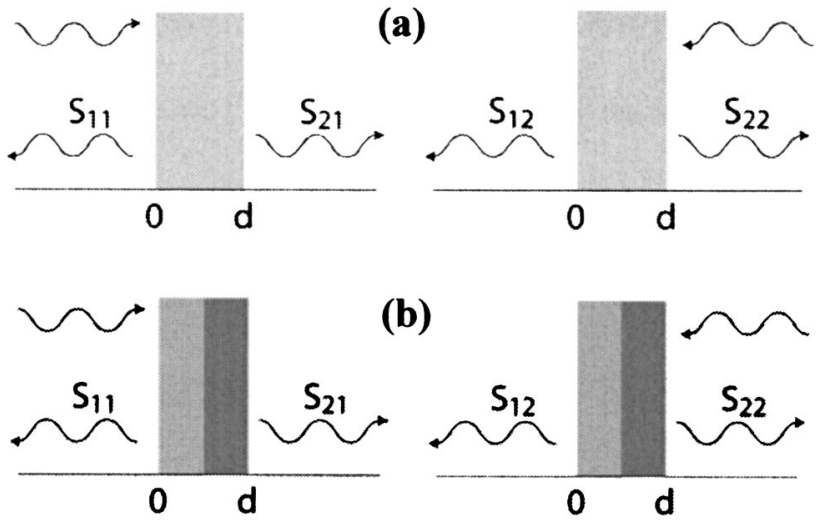

(b)
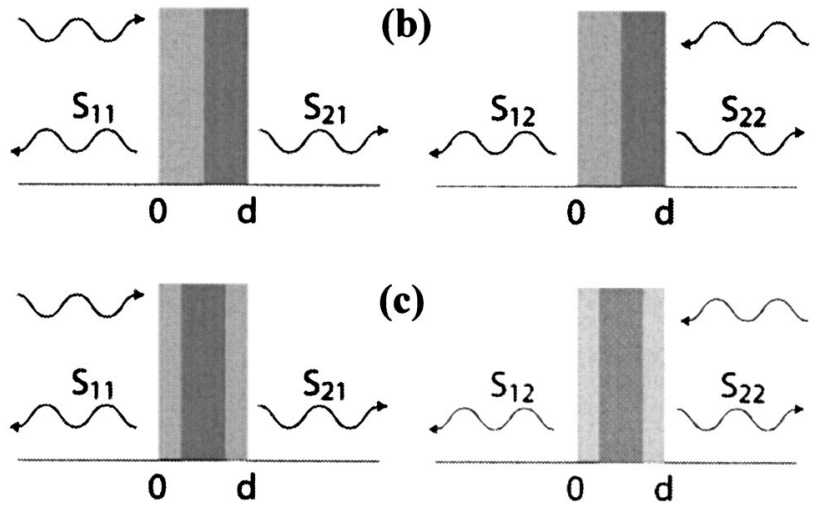

(c)

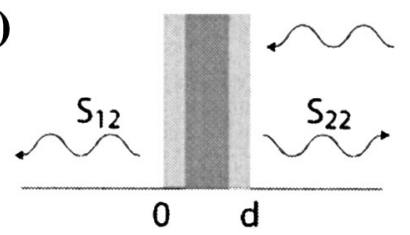

FIG. 1. $S$-parameter measurements on (a) a homogeneous 1D slab; (b) an inhomogeneous asymmetric 1D slab; and (c) a symmetric inhomogeneous 1D slab. The parameter $d$ represents the thickness of a single unit cell of the structure. The different shaded regions represent different homogeneous materials, each with distinct values of material parameters.

The procedure of $S$-parameter retrieval applied initially to metamaterial structures resulted in material parameters that were physically reasonable [18], and have been shown to be consistent with experiments on fabricated samples [19]. Yet a variety of artifacts exist in the retrieved material parameters that are related to the inherent inhomogeneity of the metamaterial. These artifacts are a result of approximating a function such as $\varepsilon(\omega, k)$ with $\varepsilon_{\text {eff }}(\omega)$; we thus expect anomalies may exist for $\varepsilon_{\text {eff }}(\omega)$. The artifacts in the retrieved material parameters are particularly severe for metamaterials that make use of resonant elements, as large fluctuations in the index and impedance can occur, such that the wavelength within the material can be on the order of or smaller than the unit cell dimension. An example of this behavior can be seen in the retrieved imaginary parts of $\varepsilon$ and $\mu$, which typically differ in sign for a unit cell that has a magnetic or an electric resonance. In homogeneous passive media, the imaginary components of the material parameters are restricted to positive values [1]. This anomalous behavior vanishes as the unit cell size approaches zero [20].

A more vexing issue for metamaterials is the finite size of the unit cell. As we will show in detail, metamaterial structures are often not conceptually reducible to the model of Fig. 1(a). It is typically the case that we must consider unit cells whose properties are more like those shown in Figs. 1(b) and 1(c) in which the equivalent unit cell consists of two (or more) distinct materials each of whose material properties differ; that is, the equivalent one-dimensional (1D) model of the material is inherently inhomogeneous, although with the assumption that the unit cell will be repeated to form a periodic medium. To understand the limitations in using the retrieval procedure on such inhomogeneous mate- rials, we must therefore understand how the effective medium and photonic crystal descriptions of periodic structures relate to the $S$-parameter retrieval procedure.

\section{B. The retrieval technique}

We outline here the general approach to the retrieval of material parameters from $S$ parameters for homogeneous materials. For the sake of generality, it is useful to first define the one-dimensional transfer matrix, which relates the fields on one side of a planar slab to the other. The transfer matrix can be defined from

$$
\mathbf{F}^{\prime}=\mathbf{T F},
$$

where

$$
\mathbf{F}=\left(\begin{array}{c}
E \\
H_{\text {red }}
\end{array}\right)
$$

$E$ and $H_{\text {red }}$ are the complex electric and magnetic field amplitudes located on the right-hand (unprimed) and left-hand (primed) faces of the slab. Note that the magnetic field assumed throughout is a reduced magnetic field [15] having the normalization $H_{\text {red }}=\left(+i \omega \mu_{0}\right) H$. The transfer matrix for a homogeneous 1D slab has the analytic form

$$
\mathbf{T}=\left(\begin{array}{cc}
\cos (n k d) & -\frac{z}{k} \sin (n k d) \\
\frac{k}{z} \sin (n k d) & \cos (n k d)
\end{array}\right),
$$

where $n$ is the refractive index and $z$ is the wave impedance of the slab [21]. $n$ and $z$ are related to $\varepsilon$ and $\mu$ by the relations

$$
\varepsilon=n / z, \quad \mu=n z .
$$

The total field amplitudes are not conveniently probed in measurements, whereas the scattered field amplitudes and phases can be measured in a straightforward manner. A scattering $(S)$ matrix relates the incoming field amplitudes to the outgoing field amplitudes, and can be directly related to experimentally determined quantities. The elements of the $S$ matrix can be found from the elements of the $T$ matrix as follows [22]:

$$
\begin{aligned}
& S_{21}=\frac{2}{T_{11}+T_{22}+\left(i k T_{12}+\frac{T_{21}}{i k}\right)}, \\
& S_{11}=\frac{T_{11}-T_{22}+\left(i k T_{12}-\frac{T_{21}}{i k}\right)}{T_{11}+T_{22}+\left(i k T_{12}+\frac{T_{21}}{i k}\right)}, \\
& S_{22}=\frac{T_{22}-T_{11}+\left(i k T_{12}-\frac{T_{21}}{i k}\right)}{T_{11}+T_{22}+\left(i k T_{12}+\frac{T_{21}}{i k}\right)},
\end{aligned}
$$




$$
S_{12}=\frac{2 \operatorname{det}(\mathbf{T})}{T_{11}+T_{22}+\left(i k T_{12}+\frac{T_{21}}{i k}\right)} .
$$

For a slab of homogeneous material, such as that shown in Fig. 1(a), Eq. (3) shows that $T_{11}=T_{22}=T_{s}$ and $\operatorname{det}(\mathbf{T})=1$, and the $S$ matrix is symmetric. Thus,

$$
\begin{aligned}
& S_{21}=S_{12}=\frac{1}{T_{s}+\frac{1}{2}\left(i k T_{12}+\frac{T_{21}}{i k}\right)}, \\
& S_{11}=S_{22}=\frac{\frac{1}{2}\left(\frac{T_{21}}{i k}-i k T_{12}\right)}{T_{s}+\frac{1}{2}\left(i k T_{12}+\frac{T_{21}}{i k}\right)} .
\end{aligned}
$$

Using the analytic expression for the $T$-matrix elements in Eq. (3) gives the $S$ parameters

$$
S_{21}=S_{12}=\frac{1}{\cos (n k d)-\frac{i}{2}\left(z+\frac{1}{z}\right) \sin (n k d)}
$$

and

$$
S_{11}=S_{22}=\frac{i}{2}\left(\frac{1}{z}-z\right) \sin (n k d) .
$$

Equations (7) and (8) can be inverted to find $n$ and $z$ in terms of the scattering parameters as follows:

$$
\begin{gathered}
n=\frac{1}{k d} \cos ^{-1}\left[\frac{1}{2 S_{21}}\left(1-S_{11}^{2}+S_{21}^{2}\right)\right], \\
z=\sqrt{\frac{\left(1+S_{11}\right)^{2}-S_{21}^{2}}{\left(1-S_{11}\right)^{2}-S_{21}^{2}}},
\end{gathered}
$$

which provide a complete material description for a slab composed of a homogeneous material. In practice, however, the multiple branches associated with the inverse cosine of Eq. (10) make the unambiguous determination of the material parameters difficult unless it is known that the wavelength within the medium is much larger than the slab length [18]. The application of Eqs. (9) and (10) to metamaterials is complicated by the minimum sample thickness set by the unit cell size. Moreover, for metamaterials based on resonant structures, there is always a frequency region over which the branches associated with the inverse cosine in Eq. (9) become very close together, and where it becomes difficult to determine the correct solution. Methods that make use of the analyticity of $\varepsilon$ and $\mu$ can be applied to achieve a more stable and accurate retrieval algorithm for these structures [23].

When the fundamental unit cell is inhomogeneous, the validity of Eqs. (9) and (10) is not clear. In particular, if the 1D material is modeled as shown in Fig. 1(b), where there is an asymmetry along the propagation direction, then $S_{11}$ and $S_{22}$ will differ, and the retrieval procedure will produce dif- ferent material parameters depending on which direction the incoming plane wave is directed. Even if the unit cell can be symmetrized, as illustrated in Fig. 1(c), the question remains as to whether the retrieval process recovers meaningful values for the material parameters. We will address these issues directly in Sec. II E below.

\section{Effective medium theory using $S$-parameter inversion}

We expect that when the scale of the unit cell is small with respect to the phase advance across it, a homogenization scheme should be applicable that will lead to averaged values for the material parameters. Since we are deriving the material parameters based on the $S$-parameter retrieval process, it is useful to demonstrate that homogenization does indeed take place within the context of this model.

Based on the model of $1 \mathrm{D}$ slabs, we note that the $T$ matrix can be expanded in orders of the optical path length $(\phi$ $=n k d)$ as follows:

$$
\mathbf{T}=\mathbf{I}+\mathbf{T}^{1} \varphi+\mathbf{T}^{2} \varphi^{2} \cdots,
$$

where $\mathbf{I}$ is the identity matrix and $\mathbf{T}^{1}$ can be found from Eq. (3) as

$$
\mathbf{T}^{1}=\left(\begin{array}{cc}
0 & -\frac{z}{k} \\
\frac{k}{z} & 0
\end{array}\right) .
$$

Using Eqs. (11) and (12) in Eq. (6), and keeping only terms to first order, we find

$$
\begin{gathered}
S_{21}=\frac{1}{1-\frac{i}{2}\left(z+\frac{1}{z}\right) \varphi}, \\
S_{11}=\frac{i}{2}\left(\frac{1}{z}-z\right) \varphi S_{21} .
\end{gathered}
$$

Equations (13) can be inverted to find approximate expressions for $n$ and $z$ analogous to Eqs. (9) and (10).

To model the effect of nonuniform unit cells, we consider a unit cell that is divided into $N$ slab regions, each with different material properties (i.e., each having an optical path length $\phi_{j}$ and impedance $z_{j}$ ), as shown in Figs. 1(b) and 1(c). The total thickness of the unit cell is then $d=d_{1}+d_{2}+\cdots$ $+d_{N}$. The transfer matrix for the composite can be found by multiplying the transfer matrix for each constituent slab, yielding

$$
\mathbf{T}=\mathbf{T}_{N} \mathbf{T}_{N-1} \cdots \mathbf{T}_{2} \mathbf{T}_{1},
$$

which, to first order, is

$$
\mathbf{T}=\mathbf{I}+\sum_{j=1}^{N} \mathbf{T}_{j}^{1} n_{j} k d_{j},
$$

with 


$$
\mathbf{T}_{j}^{1}=\left(\begin{array}{cc}
0 & -\frac{z_{j}}{k} \\
\frac{k}{z_{j}} & 0
\end{array}\right) .
$$

Using Eq. (16) in Eq. (6), we find

$$
\begin{gathered}
S_{21}=\frac{1}{1-\frac{i}{2} \sum_{j=1}^{N}\left(z_{j}+\frac{1}{z_{j}}\right) n_{j} k d_{j}}, \\
S_{11}=\frac{i}{2} \sum_{j=1}^{N}\left(\frac{1}{z_{j}}-z_{j}\right) n_{j} k d_{j} S_{21} .
\end{gathered}
$$

Comparing Eqs. (17) with Eqs. (13), we see that, to first order, we can define averaged quantities for the multicell structure as

$$
\begin{aligned}
& \left(z_{\mathrm{av}}+\frac{1}{z_{\mathrm{av}}}\right) n_{\mathrm{av}}=\sum_{j=1}^{N}\left(z_{j}+\frac{1}{z_{j}}\right) n_{j} \frac{d_{j}}{d}, \\
& \left(z_{\mathrm{av}}-\frac{1}{z_{\mathrm{av}}}\right) n_{\mathrm{av}}=\sum_{j=1}^{N}\left(z_{j}-\frac{1}{z_{j}}\right) n_{j} \frac{d_{j}}{d} .
\end{aligned}
$$

Neither $n$ nor $z$ average in a particularly simple manner; however, Eqs. (18) can be written in the physically satisfying form

$$
\begin{gathered}
\mu_{\mathrm{av}}=z_{\mathrm{av}} n_{\mathrm{av}}=\sum_{j=1}^{N} z_{j} n_{j} \frac{d_{j}}{d}=\sum_{j=1}^{N} \mu_{j} \frac{d_{j}}{d}, \\
\varepsilon_{\mathrm{av}}=\frac{n_{\mathrm{av}}}{z_{\mathrm{av}}}=\sum_{j=1}^{N} \frac{n_{j}}{z_{j}} \frac{d_{j}}{d}=\sum_{j=1}^{N} \varepsilon_{j} \frac{d_{j}}{d},
\end{gathered}
$$

which shows that the first order equations average to the expected effective medium values, in which the average permittivity is equal to the volume average of the permittivity of each component, and the average permeability is equal to the volume average of the permeability of each component. Note that in the context of this model, the homogenization limit is equivalent to requiring that the ordering of the slabs within the unit cell make no difference in the properties of the composite. From Eq. (15) we see that $\left[\mathbf{T}_{i}, \mathbf{T}_{j}\right]=O\left(\varphi_{i} \varphi_{j}\right)$, which provides a measure of the error in applying Eqs. (19) to a finite unit cell.

As we have noted, the optical path length over a unit cell for metamaterial samples is seldom such that Eqs. (19) are valid. However, if the metamaterial is composed of repeated cells, then an analysis based on periodic structures can be applied. From this analysis we may then extract some guidance as to the degree to which we might consider a periodic structure as a homogenized medium.

\section{Periodic inhomogeneous systems}

For periodic systems, no matter what the scale of the unit cell relative to the wavelength, there exists a phase advance per unit cell that can always be defined based on the periodicity. This phase advance allows the periodic structure in one dimension to be described by an index-even if effective-at all scales. We can determine the properties of a periodic structure from the $T$ matrix associated with a single unit cell. The fields on one side of a unit cell corresponding to a periodic structure are related to the fields on the other side by a phase factor, or

$$
\mathbf{F}(x+d)=e^{i \alpha d} \mathbf{F}(x),
$$

where $\mathbf{F}$ is the field vector defined in Eq. (2) and $\alpha$ is the phase advance per unit cell [21]. Equation (20) is the Bloch condition. Using Eq. (20) with Eq. (1), we have

$$
\mathbf{F}^{\prime}=\mathbf{T F}=e^{i \alpha d} \mathbf{F} .
$$

Equation (21) allows us to find the dispersion relation for the periodic medium from knowledge of the transfer matrix by solving

$$
\left|\mathbf{T}-e^{i \alpha d} \mathbf{I}\right|=0,
$$

from which we find

$$
T_{11} T_{22}-\xi\left(T_{11}+T_{22}\right)+\xi^{2}-T_{12} T_{21}=0,
$$

where we have written $\xi=\exp (i \alpha d)$. By using $\operatorname{det}(\mathbf{T})=1$, Eq. (23) can be simplified to

$$
\xi+\frac{1}{\xi}=T_{11}+T_{22},
$$

or

$$
2 \cos (\alpha d)=T_{11}+T_{22} .
$$

Note that for a binary system, composed of two repeated slabs with different material properties, $\mathbf{T}=\mathbf{T}_{1} \mathbf{T}_{2}$, and we find

$$
\begin{aligned}
\cos (\alpha d)= & \cos \left(n_{1} k d_{1}\right) \cos \left(n_{2} k d_{2}\right) \\
& -\frac{1}{2}\left(\frac{z_{1}}{z_{2}}+\frac{z_{2}}{z_{1}}\right) \cos \left(n_{1} k d_{1}\right) \cos \left(n_{2} k d_{2}\right),
\end{aligned}
$$

the well known result for a periodic 1D photonic crystal [21]. Equation (26) and, more generally, Eq. (25) provide a definition of index that is valid outside of the homogenization limit.

\section{E. Material parameter retrieval for inhomogeneous systems}

If the optical path length across the unit cell of a structure is not small, then the effective medium limit of Eqs. (19) is not applicable. Since the $S$-parameter retrieval procedure is predicated on the assumption that the analyzed structure can be replace by a continuous material defined by the parameters $n$ and $z$, it is clear that the retrieved parameters-if at all valid-will not be simply related to the properties of the constituent components. Moreover, if the unit cell is not symmetric in the propagation direction [e.g., $\varepsilon(z) \neq \varepsilon(-z)]$, then the standard retrieval procedure fails to provide a unique answer for $n$. Depending on the direction of propagation of the incident plane wave with respect to the unit cell, we find the index is defined by either 


$$
\cos (n k d)=\frac{1}{2 S_{21}}\left(1-S_{11}^{2}+S_{21}^{2}\right)
$$

or

$$
\cos (n k d)=\frac{1}{2 S_{21}}\left(1-S_{22}^{2}+S_{21}^{2}\right),
$$

which give different values since $S_{11}$ and $S_{22}$ are not the same for asymmetric structures.

However, if the unit cell is repeated infinitely, Eq. (25) shows that a unique value of index can be recovered. To compare the form of Eq. (25) with Eqs. (27) and (28), we first express the $S$-matrix elements in terms of the $T$-matrix elements as follows [22]:

$$
\begin{aligned}
& T_{11}=\frac{\left(1+S_{11}\right)\left(1-S_{22}\right)+S_{21} S_{12}}{2 S_{21}}, \\
& T_{12}=\frac{\left(1+S_{11}\right)\left(1+S_{22}\right)-S_{21} S_{12}}{2 S_{21}}, \\
& T_{21}=\frac{\left(1-S_{11}\right)\left(1-S_{22}\right)-S_{21} S_{12}}{2 S_{21}}, \\
& T_{22}=\frac{\left(1-S_{11}\right)\left(1+S_{22}\right)+S_{21} S_{12}}{2 S_{21}} .
\end{aligned}
$$

Using Eqs. (29) in Eq. (25) yields

$$
\cos (\alpha d)=\frac{1-S_{11} S_{22}+S_{21}^{2}}{2 S_{21}}
$$

Equation (30) shows that, regardless of the wavelength to unit cell ratio, an effective index can be recovered from a modified $S$-parameter retrieval procedure that utilizes all elements of the $S$ matrix. A comparison of Eq. (30) with Eqs. (27) and (28) shows that the standard retrieval procedure can be applied to find the index for an inhomogeneous structure using an averaged $S_{\text {av }}$ of

$$
S_{\mathrm{av}}=\sqrt{S_{11} S_{22}} .
$$

The analysis that leads to Eq. (30) does not require any assumption that the unit cell size be negligible in comparison to the optical path length. As such, the retrieved index will be valid well beyond the regime where traditional effective medium theory would be valid, in agreement with [11]. This result explains the apparent success in assigning bulk material parameters to metamaterial structures. Note that the retrieval procedures for both homogeneous and symmetric inhomogeneous unit cells are identical, since $S_{11}=S_{22}$.

To obtain values for $\varepsilon$ and $\mu$, it is also necessary to determine the impedance $z_{\text {red }}$ for the inhomogeneous medium. For a continuous material, $z_{\text {red }}$ is an intrinsic parameter that describes the ratio of the electric to the magnetic fields $\left(E / H_{\text {red }}\right)$ for a plane wave. Clearly, for an inhomogeneous material this ratio will vary throughout the unit cell leading to an unavoidable ambiguity in the definition of $z_{\text {red }} \cdot z_{\text {red }}$ can be found from Eq. (21) as

$$
z_{\text {red }}=\frac{T_{12}}{T_{11}-\xi}
$$

or, equivalently,

$$
z_{\text {red }}=\frac{T_{22}-\xi}{T_{21}} .
$$

Solving Eq. (23) for $\xi$ yields

$$
\xi=\frac{\left(T_{11}+T_{22}\right)}{2} \pm \sqrt{\frac{\left(T_{22}-T_{11}\right)^{2}+4 T_{12} T_{21}}{4}} .
$$

On substitution of Eq. (34) into Eq. (33) [or Eq. (32)] we obtain two results for $z_{\text {red }}$ corresponding to the two eigenvalues of Eq. (23):

$$
z_{\text {red }}=\frac{\left(T_{22}-T_{11}\right) \mp \sqrt{\left(T_{22}-T_{11}\right)^{2}+4 T_{12} T_{21}}}{2 T_{21}} .
$$

The two roots of this equation correspond to the two directions of wave propagation. Inspection of Eq. (35) reveals that for a reciprocal structure, $T_{11}=T_{22}$ and the impedance has the form

$$
z_{\text {red }}^{2}=\frac{T_{12}}{T_{21}} .
$$

Using the values for the $T$-matrix elements, Eqs. (29), we recover the same form for $z_{\text {red }}$ as in Eq. (10).

Although an inhomogeneous periodic structure does not have a well defined impedance, as the ratio of $E / H_{\text {red }}$ will vary periodically throughout the structure, this variation becomes negligible for very small unit cell sizes relative to the wavelength. Nevertheless, the lack of a unique definition for $z_{\text {red }}$ indicates that the values of $\varepsilon$ and $\mu$ retrieved are not generally assignable, although they can be applied in an artificial manner if the metamaterial is always to be terminated in the same location of the unit cell. The surface termination has an increasing influence on the scattering properties of the structure as the scale of inhomogeneity increases relative to the wavelength.

\section{NUMERICAL EXAMPLES}

\section{A. $S$-parameter retrieval for a symmetric structure}

We illustrate some of the issues associated with the retrieval procedure by using $S$ parameters obtained from numerical simulations on negative index metamaterials. Negative index metamaterials, which have been of recent interest, pose a significant challenge to retrieval methods because they utilize resonant elements and exhibit both an electric and magnetic response. A single unit cell of a typical symmetric (in the propagation direction) metamaterial structure is shown in Fig. 2. This particular structure is composed of two types of conducting elements-a split ring resonator (SRR) and a wire - that have been designed to yield a band of negative refractive index at microwave frequencies. Similar structures have now been analyzed using $S$-parameter retrieval methods by numerous authors [18-20,23], with the general observation that physically reasonable results can be obtained. 

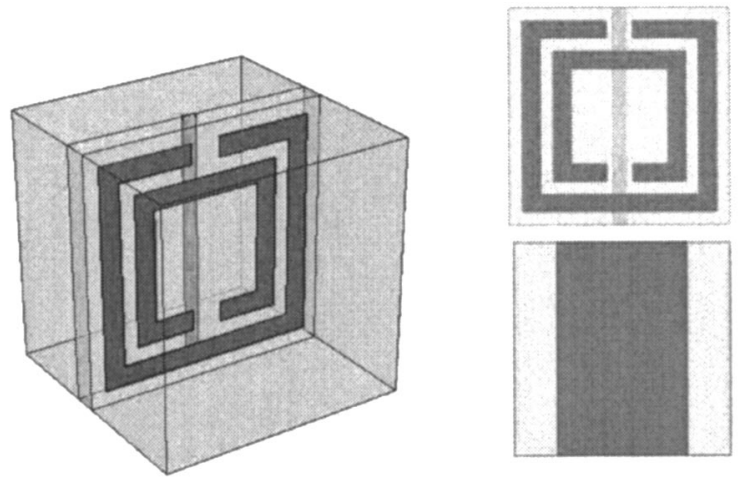

FIG. 2. (Left) A single unit cell of a negative index metamaterial. The split ring resonator (SRR) responds magnetically and the wire responds electrically to electromagnetic fields. (Right, top) Planar view of the unit cell. Wave propagation is from left to right. (Right, bottom) An equivalent model of the unit cell, schematically indicating that it is a symmetric but inhomogeneous structure.

We note in passing that the SRR generally exhibits bianisotropy, since an applied magnetic field induces both an electric as well as a magnetic response. This material response should properly be accounted for in a complete characterization procedure. However, for the orientation of the SRR shown in Fig. 2 (ring gaps in the direction of the electric field), previous work has indicated that the crosscoupling terms can be negligible, so that we are justified here in pursuing a description of the composite in terms of only $\varepsilon$ and $\mu$.

The details of the retrieval procedure for metamaterials have been outlined previously in [18], with a more stable implementation described in [23]. The geometry and dimensions for the present example were chosen to be roughly consistent with previous studies. The unit cell is cubic, with a cell dimension of $d=2.5 \mathrm{~mm}$. A $0.25 \mathrm{~mm}$ thick substrate of FR4 ( $\varepsilon=4.4$, loss tangent of 0.02$)$ is assumed. A copper SRR and wire are positioned on opposite sides of the substrate, modeling the structures typically produced by lithographic circuit board techniques. The copper thickness is $0.017 \mathrm{~mm}$. The width of the wire is $0.14 \mathrm{~mm}$, and it runs the length of the unit cell. The outer ring length of the SRR is $2.2 \mathrm{~mm}$ and both rings have a linewidth of $0.2 \mathrm{~mm}$. The gap in each ring is $0.3 \mathrm{~mm}$, and the gap between the inner and outer rings is $0.15 \mathrm{~mm}$.

The $S$ parameters for the unit cell of Fig. 2 are computed using HFSS (Ansoft), a commercial finite-element-based electromagnetic mode solver. Both the $S$ parameters and the retrieved material parameters are presented in Fig. 3. Figures 3(a) and 3(b) show the magnitude and phase of the computed $S$ parameters. Note the dip in the phase of $S_{21}$, which indicates the presence of a negative index band.

The retrieved index in Fig. 3(c) confirms the negative index band that lies between roughly 9 and $12 \mathrm{GHz}$. The structure was designed so as to be roughly impedance matched $(z=1)$ where $n=-1$, as this material condition is desirable for many applications $[24,25]$. The retrieved impedance, shown in Fig. 3(d), shows that the structure is indeed roughly matched at the frequency where $n=-1$. Using the impedance and index in Eq. (4), we find functional forms


FIG. 3. (a) Magnitude and (b) phase of the simulated $S$ parameters for the unit cell in Fig. 2. Retrieved index (c), impedance (d), permittivity (e), and permeability (f) are also shown.

for the permittivity and permeability, as shown in Figs. 3(e) and 3(f).

While the retrieval procedure leads to generally satisfying results-an expected resonance function for $\mu$, for example-unphysical artifacts occur in the recovered mate- 

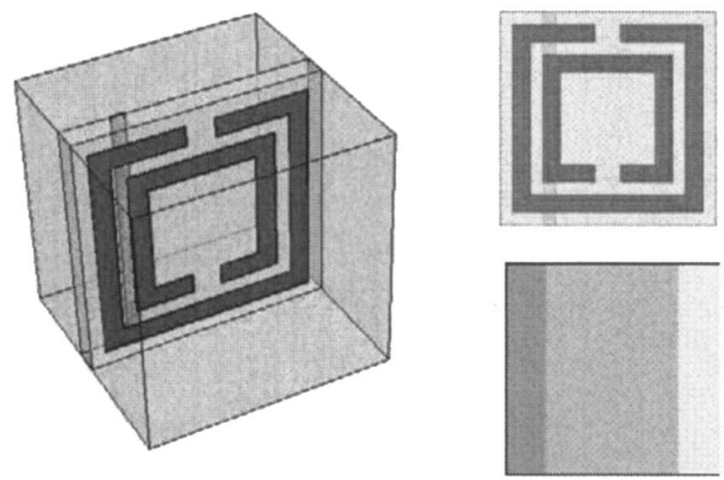

FIG. 4. An asymmetric unit cell. The parameters of the SRR and wire are the same as in the unit cell depicted in Fig. 2, except that the wire has been shifted $0.75 \mathrm{~mm}$ away from its centered position. The equivalent model (bottom, right) depicts the asymmetry of the structure.

rial parameters suggesting that a homogenization limit has not been reached. Note, for example, that there is a slight antiresonance in the real part of $\varepsilon$ near $9 \mathrm{GHz}$ [Fig. 3(e)], which is consistent with the imaginary part of $\varepsilon$ being negative at the same frequencies. This artifact can be recovered by replacing the actual SRR-wire unit cell with the inhomogeneous unit cell indicated in Fig. 2(c). The inhomogeneous unit cell consists of three slabs, the center slab of which contains a material with idealized frequency-dependent $\varepsilon$ and $\mu$. The outer slabs are uniform material (vacuum or dielectric, for example). The details of how this type of inhomogeneity leads to artifacts and distortions in the retrieved material parameters have been recently described [20], and will not be repeated here, as a more detailed analysis is forthcoming [26]. For the structure presented in Fig. 2, the anomalies are relatively minor, and the retrieved material parameters provide somewhat accurate descriptions of the electromagnetic material properties.

Note that while we appeal to the slab model of Sec. II in our analysis here and in the following sections, we do not attempt to solve for the effective slab parameters or thicknesses, as this would constitute a nontrivial inverse problem, and is not of direct relevance here as we are searching for a homogenized description of the composite medium.

\section{B. $S$-parameter retrieval for a nonsymmetric structure}

The unit cell in Fig. 2 can be made asymmetric by moving the wire, for example, off of the symmetry axis. The unit cell shown in Fig. 4 is identical to that of Fig. 2, except that the wire has been shifted a distance of $0.75 \mathrm{~mm}$ along the propagation direction. Figures 5(a) and 5(b) show the $S$ parameters computed for an infinitely repeated asymmetric unit cell, one cell thick in the direction of propagation. While the differences between the magnitudes of $S_{11}$ and $S_{22}$ are modest, there is a great contrast in the phases of $S_{11}$ and $S_{22}$, implying very different impedance properties for the structure depending on which side of the unit cell faces the incoming wave (both the phases and magnitudes of $S_{12}$ and $S_{21}$ are identical). The standard retrieval procedure to find the index [Eqs. (27)


FIG. 5. (a) Magnitude and (b) phase of the simulated $S$ parameters for the unit cell in Fig. 4. (c) The retrieved refractive index, using the standard retrieval method (gray curves) and the full $S$ parameters (solid black curve). (d) A comparison of the refractive indices for the symmetric and asymmetric unit cells. (e) The two impedance functions $z_{1}$ and $z_{2}$ corresponding to the two different propagation directions.

and (28)] yields the unphysical functional forms shown by the solid and dashed gray lines in Fig. 5(c). Using the modified $S$-parameter retrieval procedure [Eq. (30)], however, yields a frequency dependent form for the index nearly identical to that of the symmetric unit cell, as shown by the solid black line in Fig. 5(c).

The retrieved indices for the symmetric and asymmetric unit cells are compared in Fig. 5(d), illustrating that, aside from a shift in frequency, the refractive properties of the two 

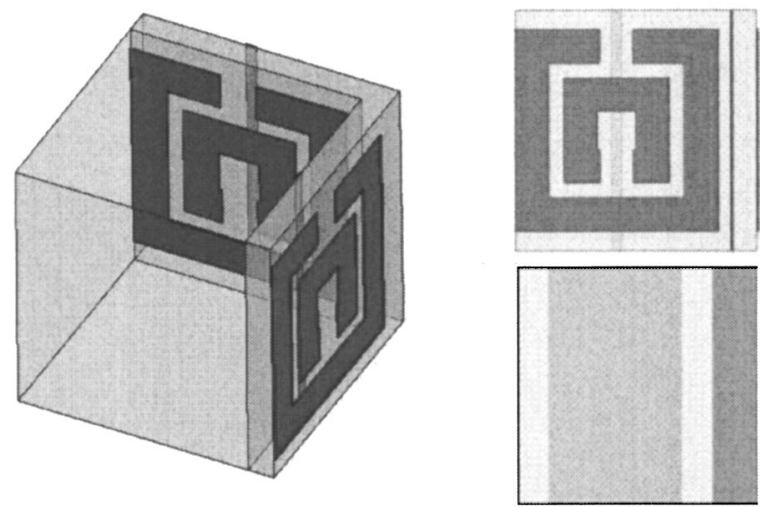

FIG. 6. A higher-dimensional structure, designed to have a frequency where $n=-1$ and $z=1$. The unit cell is asymmetric in any direction of propagation within the plane. An equivalent model of the unit cell is shown (bottom, right).

structures are very similar. The frequency shift is not surprising, as there is likely an interaction between the SRR and wire that leads to somewhat different material properties depending on their relative positions. The effect of this interaction, as evidenced from Fig. 5(d), is relatively minor.

While the index is nearly the same for the structures having symmetric and asymmetric unit cells, the impedance is clearly different, as indicated in Fig. 5(e) [cf. Fig. 3(d)]. So different are the two solutions for $z$ for the asymmetric structure that in general the assignment of values of $\varepsilon$ and $\mu$ to the composite becomes counterproductive. Thus, although a well-defined refractive index exists for the composite, the manner in which a wave scatters from this material can depend strongly on how the surface is terminated.

Figure 5(e) shows that the two solutions for the impedance found from Eq. (35), although very different, can actually have similar values at frequencies removed from either the resonance or the effective plasma frequency where $\operatorname{Re}(\varepsilon)=0$ (at $\sim 14 \mathrm{GHz}$ ). Given that the refractive index is well defined, at frequencies where the two impedance solutions are the same the asymmetric unit cell also mimics a homogenized material, although the material properties are somewhat different from those retrieved from the symmetric unit cell. The asymmetric structure, for example, is not impedance matched where $n=-1$ as is the symmetric structure.

\section{Higher-dimensional metamaterial structures}

The issue of symmetry does not arise for most 1D structures, since the unit cell can usually be made symmetric along the propagation axis. However, for structures in which it is desired to tune the electromagnetic properties in more than one dimension, the resulting unit cell may be unavoidably asymmetric. While higher-dimensional symmetric structures have been proposed that can serve as the basis for isotropic negative index metamaterials [27], construction of such structures may not always be feasible for the fabrication methods most readily available.

A simple method of making the SRR-wire unit cell approximately isotropic within a plane of propagation (and for a given polarization) is to add a second SRR-wire structure
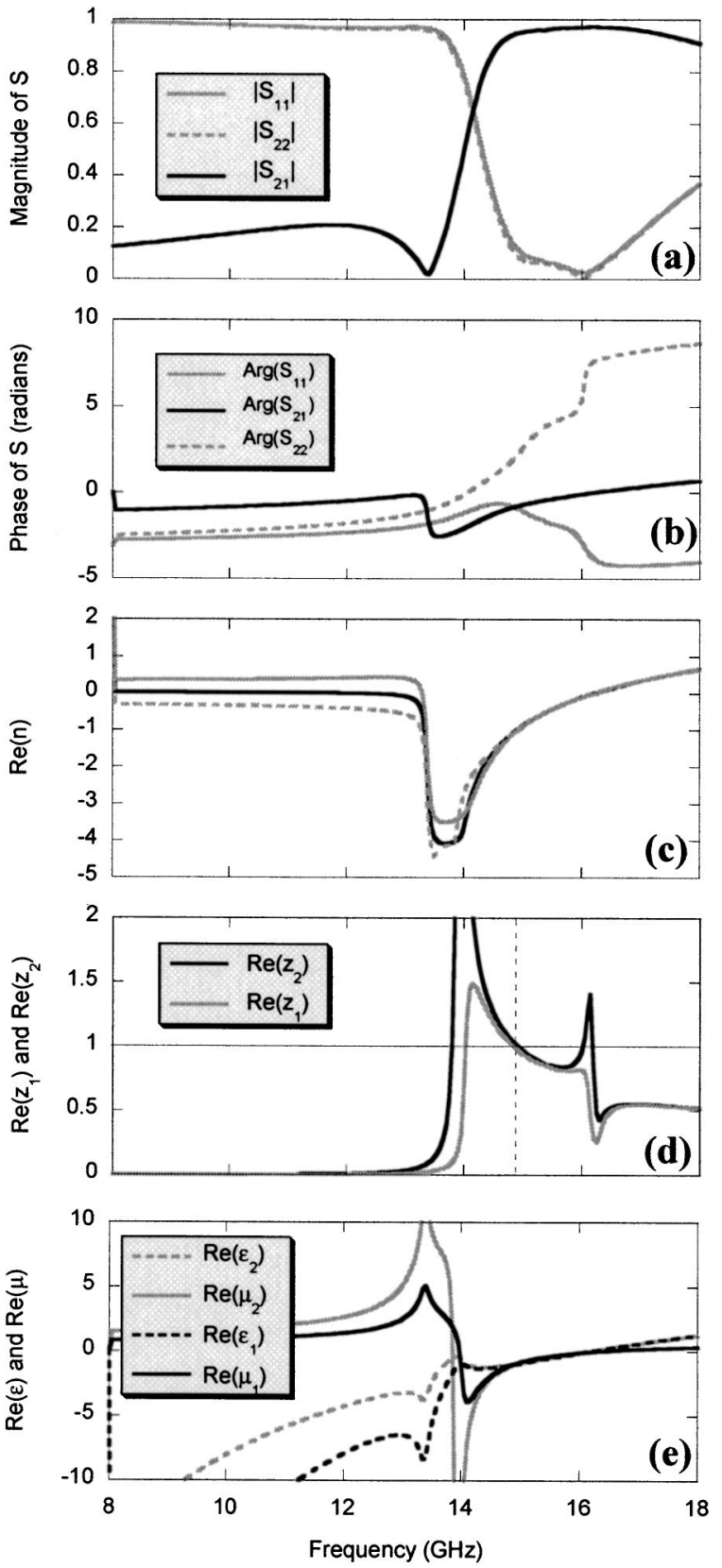

FIG. 7. (a) Magnitude and (b) phase of the simulated $S$ parameters for the unit cell in Fig. 2. (c) The retrieved index using the standard retrieval method (gray curves) and the full $S$ parameter method (solid black curve). (d) The impedance values are shown for both directions of incidence on the unit cell, as are the permittivity and permeability (e).

within the unit cell, oriented perpendicular to the first SRR. An example unit cell of this type is shown in Fig. 6. This method of approximating isotropy has been used in previously demonstrated negative index metamaterials based on circuit board fabrication, in which strips of patterned circuit board are assembled together in a wine crate structure [13]. Note, however, that this sample must be considered as inhomogeneous, given the wavelengths of operation relative to size of the unit cell, and must be analyzed in the same manner as the asymmetric unit cell above. 
The unit cell for this structure is the same size as in the previous examples $(2.5 \mathrm{~mm})$, but has different parameters for the SRRs, substrate, and wires. A $0.25 \mathrm{~mm}$ substrate of Duroid circuit board (Rogers, $\varepsilon=2.2$, loss tangent of 0.0009) is assumed. A copper SRR and wire are positioned on opposite sides of both perpendicularly positioned circuit boards. The copper thickness is $0.017 \mathrm{~mm}$. The width of the wire is $0.10 \mathrm{~mm}$, and it runs the length of the unit cell. The outer ring length of the SRR is $2.2 \mathrm{~mm}$ and both rings have a linewidth of $0.35 \mathrm{~mm}$. The gap in each ring is $0.35 \mathrm{~mm}$, and the gap between the inner and outer rings is $0.15 \mathrm{~mm}$. The dimensions of the rings and wires of this structure were carefully tuned to the above values, so that the structure would be matched where $n=-1$.

The retrieved parameters for the 2D unit cell of Fig. 6 are shown in Fig. 7. The frequency dependent forms for $\operatorname{Re}(\mu)$ and $\operatorname{Re}(\varepsilon)$ are shown in Fig. 7(e). At frequencies above $15 \mathrm{GHz}$, the material parameters retrieved for waves traveling in opposite directions are fairly similar, even though they disagree in general over the broader frequency range. Near $15 \mathrm{GHz}$, both retrieved values of $z$ are nearly equal to unity while the refractive index is near -1 , so that the structure responds as if it were approximately homogeneous at that frequency. Note that for this asymmetric 2D structure, similar to the example of Fig. 5(c), the refractive index determined from the modified retrieval method is quite different from that determined from the standard retrieval method. This example further illustrates the necessity of using the modified full $S$-parameter retrieval method for asymmetric structures.

\section{CONCLUSION}

We have shown that metamaterials based on periodic structures occupy conceptually a special niche between effective media and photonic crystals. The $S$-parameter retrieval techniques that have been utilized recently to characterize metamaterials have been shown to be valid for metamaterials having symmetric unit cells, even when the optical path length is on the order of the unit cell size. For asymmetric unit cells, a modified retrieval method leads to a unique value of the refractive index, but two generally distinct values for the wave impedance that are assigned to the structure depending on the direction of wave propagation with respect to the unit cell. The general lack of uniqueness of $z$ complicates but does not obviate the design of structures or devices that are necessarily asymmetric, since frequency regions can be found where the two values of $z$ are nearly equal. Nevertheless, the expanded analysis presented here is required to determine whether or not an inhomogeneous structure can be approximated as a homogeneous metamaterial.

\section{ACKNOWLEDGMENTS}

This work was supported by DARPA (Contract No. MDA972-01-2-0016) and DARPA/ONR through a MURI program (Contract No. N00014-01-1-0803). The work of C.M.S. is further supported by the Ames Laboratory (Contract No. W-7405-Eng-82) and EU-FET project DALHM. The authors are grateful to Dr. Tomasz Grzegorczyk for extensive comments and discussion.
[1] L. D. Landau, E. M. Lifshitz, and L. P. Pitaevskii, Electrodynamics of Continuous Media, 2nd ed. (Butterworth-Heinenann, Oxford, England, 1984).

[2] D. J. Bergman, Phys. Lett., C 43, 377 (1978).

[3] V. M. Shalaev, Phys. Rep. 272, 61 (1996).

[4] K. Bao, R. C. McPhedran, N. A. Nicorovici, C. G. Poulton, and L. C. Botten, Physica B 279, 162 (2000).

[5] C. Grimes and D. M. Grimes, Phys. Rev. B 43, 10780 (1991).

[6] J. A. Kong, Electromagnetic Wave Theory, 2nd ed. (John Wiley \& Sons, New York, 1990).

[7] N. Engheta, J. Electromagn. Waves Appl. 6, 1 (1992).

[8] J. B. Pendry, A. J. Holden, D. J. Robbins, and W. J. Stewart, IEEE Trans. Microwave Theory Tech. 47, 2075 (1999).

[9] D. R. Smith, D. C. Vier, N. Kroll, and S. Schultz, Appl. Phys. Lett. 77, 2246 (2000).

[10] A. K. Sarychev, R. C. McPhedran, and V. M. Shalaev, Phys. Rev. B 62, 8531 (2000).

[11] C. A. Kyriazidou, H. F. Contopanagos, W. M. Merrill, and N. G. Alexópoulos, IEEE Trans. Antennas Propag. 48, 95 (2000).

[12] D. R. Smith, W. J. Padilla, D. C. Vier, S. C. Nemat-Nasser, and S. Schultz, Phys. Rev. Lett. 84, 4184 (2000).
[13] R. Shelby, D. R. Smith, and S. Schultz, Science 292, 77 (2001).

[14] K. M. Ho, C. T. Chan, and C. M. Soukoulis, Phys. Rev. Lett. 65, 3152 (1991).

[15] J. B. Pendry, J. Phys.: Condens. Matter 8, 1085 (1996).

[16] W. B. Weir, Proc. IEEE 62, 33 (1974).

[17] A. M. Nicholson and G. F. Ross, IEEE Trans. Instrum. Meas. IM-19, 377 (1970).

[18] D. R. Smith, S. Schultz, P. Markoš, and C. M. Soukoulis, Phys. Rev. B 65, 195104 (2002).

[19] A. F. Starr, P. M. Rye, D. R. Smith, and S. Nemat-Nasser, Phys. Rev. B 70, 115113 (2004).

[20] T. Koschny, P. Markoš, D. R. Smith, and C. M. Soukoulis, Phys. Rev. E 58, 065602 (2003).

[21] D. R. Smith, R. Dalichaouch, N. Kroll, S. Schultz, S. L. McCall, and P. M. Platzman, J. Opt. Soc. Am. B 10, 314 (1993).

[22] D. M. Pozar, Microwave Engineering, 2nd ed. (John Wiley \& Sons, New York, 1998), p. 211.

[23] X. Chen, T. M. Grzegorczyk, B.-I. Wu, J. Pacheco, and J. A. Kong, Phys. Rev. E 70, 016608 (2004).

[24] J. B. Pendry, Phys. Rev. Lett. 85, 3966 (2000). 
[25] C. G. Parazzoli, R. B. Greegor, J. A. Nielsen, M. A. Thompson, K. Li, A. M. Vetter, M. H. Tanielian, and D. C. Vier, Appl. Phys. Lett. 84, 3232 (2004).

[26] T. Koschny, P. Markos, E. N. Economou, D. R. Smith, D. C.
Vier, and C. M. Soukoulis, (unpublished).

[27] P. Gay-Balmaz and O. J. F. Martin, Appl. Phys. Lett. 81, 639 (2002). 